\title{
Systematic Review and Meta-analysis of Postlicensure Observational Studies on Human Papillomavirus Vaccination and Autoimmune and Other Rare Adverse Events
}

\author{
Corinne Willame, MPH, * Kartini Gadroen, MD, † Wichor Bramer, BSc, $\neq$ Daniel Weibel, PhD, $†$ \\ and Miriam Sturkenboom, PhD*
}

\begin{abstract}
Background: Because of the limited number of subjects in prelicensure studies, autoimmune diseases and other rare adverse effects of vaccines may go undetected. Since 2006, millions of human papillomavirus (HPV) vaccine doses have been distributed and a considerable amount of postlicensure safety data has been generated. The objective of this study was to review available HPV postlicensure safety studies and to summarize risk estimates of autoimmune and other rare diseases.

Methods: For this systematic review and meta-analysis, we searched literature databases to identify any postlicensure safety studies related to HPV vaccination and autoimmune adverse events from inception to April 16, 2019. Pooled risk estimates were computed using fixed- or randomeffects models if at least 2 estimates per disease and per HPV vaccine were available

Results: Twenty-two studies met our inclusion criteria. The studies applied various methodologies and used different types of data sources and outcome definitions. Quadrivalent HPV vaccine (4vHPV) was most commonly assessed. Type 1 diabetes mellitus, immune thrombocytopenia purpura and thyroiditis diseases were most frequently reported. The meta-analysis was conducted on 35 diseases corresponding to 48 pooled risk estimates. Majority of the pooled estimates showed no significant effect $(n=43)$. Three negative (paralysis, immune thrombocytopenia purpura and chronic fatigue syndrome) and 2 positive (Hashimoto and Raynaud diseases) associations were detected.

Conclusion: Our study demonstrated an absence of clear association between HPV vaccines and autoimmune and other rare diseases. The review also highlights the need for more systematic collaborations to monitor rare safety adverse events
\end{abstract}

Key Words: papillomavirus vaccines, autoimmune diseases, rare adverse events, postlicensure studies

(Pediatr Infect Dis J 2020;39:287-293)

Accepted for publication November 29, 2019.

From the *Julius Center for Health Sciences and Primary Care, University Medical Center, Utrecht, the Netherlands; $\dagger$ Department of Medical Informatics, Erasmus MC, University Medical Center, Rotterdam, the Netherlands; and $\ddagger$ Medical Library, Erasmus MC, University Medical Center, Rotterdam, the Netherlands.

C.W. works part time with the GSK group of companies, and she declares not to receive any financial support from the GSK group of companies for the submitted work. M.S. is coordinator of the ADVANCE consortium, and until 2016 she ran a research group that conducted research for pharmaceutical industries according to European Network of Centres for Pharmacoepidemiology and Pharmacovigilance code of conduct, none related to this topic. D.W. is a consultant for GSK regarding malaria vaccine safety studies. The other authors have no funding or conflicts of interest to disclose.

Address for correspondence: Corinne Willame, MPH, University Medical Center Utrecht, Julius Center for Health Sciences and Primary Care, PO Box 85500, 3508 GA Utrecht, the Netherlands. E-mail: c.willame@umcutrecht.nl.

Supplemental digital content is available for this article. Direct URL citations appear in the printed text and are provided in the HTML and PDF versions of this article on the journal's website (www.pidj.com).

Copyright (C) 2019 Wolters Kluwer Health, Inc. All rights reserved.

ISSN: 0891-3668/20/3904-0287

DOI: $10.1097 /$ INF.0000000000002569
$\mathrm{H}$ uman papillomavirus (HPV) vaccines are effective in reducing HPV infections ${ }^{1,2}$ and in preventing cervical cancer, caused by certain HPV genotypes. ${ }^{3}$ Currently 3 licensed HPV vaccines are available: a quadrivalent HPV vaccine (4vHPV; Gardasil, Merck, USA) and a bivalent HPV vaccine (2vHPV; Cervarix, GSK, Belgium) licensed in 2006 and 2007, respectively, followed in late 2014 by 9 -valent HPV vaccine (9vHPV; Gardasil9, Merck, USA). Table 1 summarizes the characteristics of the 3 vaccines. All 3 HPV vaccines were initially licensed and marketed using a 3 -dose vaccination schedule. However, a 2-dose schedule was subsequently approved for all 3 vaccines. HPV vaccines are available in more than 100 countries, ${ }^{4}$ and over 80 countries have included the HPV vaccine into their national immunization programs mainly targeting young adolescent girls. ${ }^{5}$ As of 2014, it is estimated that 59 million women have received at least 1 dose of HPV vaccine. ${ }^{6}$ Routine vaccination of boys is currently implemented in several countries worldwide.

To enhance the immune response, HPV vaccines contain adjuvanted systems, such as toll-like receptors or oil-based emulsions. These adjuvant vaccines enhance a general immune response and may potentially trigger autoimmune reactions (responses against body's own tissue). ${ }^{7}$ The safety of vaccines and its adjuvants require assessment pre licensure and continuous monitoring post licensure. This is done by passive surveillance of case reports and active surveillance studies, aiming to detect rare reactions or associations with diseases that have low incidences. As part of postmarketing commitments and requests, several studies were conducted aiming to estimate the risks of developing autoimmune diseases following HPV vaccination. ${ }^{8,9}$ To date, available meta-analysis of HPV vaccine and autoimmune diseases have largely synthesized results of clinical trials ${ }^{10}$ or a mix of postlicensure studies and clinical trials. ${ }^{11}$ To address the need to analyze available postlicensure safety data, we carried out a systematic review of postlicensure observational safety studies assessing the risk of autoimmune and rare adverse events following HPV vaccination; we describe the methodologic approaches used and we summarize the risk estimates.

\section{MATERIALS AND METHODS}

We used a comprehensive 3-step search strategy to identify relevant studies. No language restrictions were placed on the searches or search results. The study conforms to the Preferred Reporting Items for Systematic reviews and Meta-analysis guidelines $^{12}$ and European Network of Centres for Pharmacoepidemiology and Pharmacovigilance ${ }^{13}$ (ENCePP) guidelines.

\section{Search Strategy and Selection Criteria}

First, we searched Embase.com, Medline (Ovid), ISI Web of Science and Cochrane Central from inception to April 16, 2019, for any postlicensure observational safety studies assessing the risk of autoimmune adverse events following HPV vaccination. A search strategy was developed for each database with a combination of free 
TABLE 1. Characteristics of HPV Vaccines

\begin{tabular}{|c|c|c|c|c|}
\hline & & $\begin{array}{l}\text { Cervarix (Recombinant, } \\
\text { Adjuvanted, Adsorbed) }\end{array}$ & $\begin{array}{c}\text { Gardasil } \\
\text { (Recombinant, Adsorbed) }\end{array}$ & $\begin{array}{c}\text { Gardasil9 } \\
\text { (Recombinant, Adsorbed) }\end{array}$ \\
\hline \multicolumn{2}{|c|}{ Recommended route of administration } & Intramuscular & Intramuscular & Intramuscular \\
\hline \multirow{5}{*}{$\begin{array}{l}\text { Characteristics by } \\
\text { dose }(0.5 \mathrm{~mL})\end{array}$} & Formulation & Suspension & Suspension & Suspension \\
\hline & $\begin{array}{l}\mathrm{L} 1 \text { virus-like particle } \\
\text { types }\end{array}$ & HPV-16 $(20 \mu \mathrm{g}), 18(20 \mu \mathrm{g})$ & $\begin{array}{l}\text { HPV-6 }(20 \mu \mathrm{g}), 11(40 \mu \mathrm{g}), 16 \\
\quad(40 \mu \mathrm{g}), 18(20 \mu \mathrm{g})\end{array}$ & $\begin{array}{l}\text { HPV-6 (30 } \mu \mathrm{g}), 11(40 \mu \mathrm{g}), 16 \\
\quad(60 \mu \mathrm{g}), 18(40 \mu \mathrm{g}), 31(20 \mu \mathrm{g}), \\
\quad 33(20 \mu \mathrm{g}), 45(20 \mu \mathrm{g}), 52(20 \\
\mu \mathrm{g}), 58(20 \mu \mathrm{g})\end{array}$ \\
\hline & Cross-protection & HPV-31, HPV-33, HPV-45 & HPV-31 & None \\
\hline & Adjuvant & $\begin{array}{l}\text { Adsorbed on aluminum hydroxide, } \\
\text { hydrated }(\mathrm{Al}(\mathrm{OH}) 3)(0.5 \mathrm{mg}) \mathrm{Al} 3+ \\
\text { in total } \\
\text { Adjuvanted by AS04 containing: } \\
\text { 3--desacyl-4'-MPL }(50 \mu \mathrm{g})\end{array}$ & $\begin{array}{l}\text { Adsorbed on amorphous alu- } \\
\text { minum hydroxyphosphate } \\
\text { sulfate adjuvant }(0.225 \mathrm{mg} \mathrm{Al})\end{array}$ & $\begin{array}{l}\text { Adsorbed on amorphous alu- } \\
\text { minum hydroxyphosphate } \\
\text { sulfate adjuvant }(0.5 \mathrm{mg} \mathrm{Al})\end{array}$ \\
\hline & Expression system & Baculovirus-insect cell & Yeast cells & Yeast cells \\
\hline
\end{tabular}

MPL, monophosphoryl lipid A.

text and controlled vocabulary (ie, medical subject headings terms). Additional search terms were included in consultation with a reference librarian (WB). The detailed search strategies for each database are presented in Table, Supplemental Digital Content 1, http://links. lww.com/INF/D738. Second, we screened reference lists of publications retrieved to identify additional relevant studies. Third, we searched web-based platforms including the EnCePP register ${ }^{14}$ and manual searches in health authorities' websites and Google Scholar.

Two reviewers $(\mathrm{CW}, \mathrm{KG})$ independently screened titles and abstracts followed by the retrieval and reviewed full-text articles according to the predefined eligibility criteria described below. Disagreements were resolved through discussion. The following inclusion criteria were applied: (1) HPV vaccination; (2) postlicensure studies; (3) epidemiologic or Phase IV studies; (4) healthy population and (5) risk/safety assessment. Commentaries, meeting reports, letters to editors, case reports, biologic or animal studies were excluded. Eligible papers, as well as papers which could not be excluded right away, were then included in the full-text assessment. Selected papers were assessed by reviewing the full-text according to the following inclusion criteria: (1) HPV-related adverse events, (2) autoimmune diseases and rare safety outcomes assessment, (3) no assessment using spontaneous reporting database.

\section{Data Abstraction and Quality Assessment}

For each of the eligible studies, we extracted the following data as a minimum: first author and year of publication, study design, objective and period, data source, geographical area, age of subjects, type of vaccine, adverse events reported either as individual or composite endpoints, method for identification and validation of cases, disease onset, risk window and risk estimates.

The study quality was assessed by the 2 same reviewers using an adapted quality checklist combining the Newcastle-Ottawa Scale ${ }^{15}$ and the Scottish Intercollegiate Guidelines Network ${ }^{16}$ quality criteria for cohort and case-control studies. The adapted quality checklist, including assessment of case-only design, is presented in Table, Supplemental Digital Content 2, http://links.lww.com/INF/D739. To assess the quality of the studies, we scored each of the studies following 4 parameters: the selection of study groups (2 points), confounding factors ( 1 point), assessment of the outcome ( 3 points) and assessment of the exposure ( 1 point). The total score represented the sum of scores for each parameter. This score was used as a relative measure of data quality; no threshold for exclusion was applied.

\section{Data Analysis}

Pooled risk estimates (odds ratios, ORs) and their respective 95\% confidence intervals (CIs) for individual autoimmune diseases were calculated if at least 2 risk estimates per outcome and per HPV vaccine type were available. Under the rare disease assumption, the OR and relative risk (RR) can be treated as approximately equal. Therefore, the pooled estimate was computed by pooling any risk estimates, independently of the type of risk measurement (relative risk, OR or hazard ratio) and by using fixed-effects model (MantelHaenszel method) or random-effects model (Der Simonian-Laird method). To determine the extent of variation between studies, we computed heterogeneity tests with Higgins $I^{2}$ statistic to measure the proportion of observed variance that reflects true effect sizes. $I^{2}$ values over $50 \%$ were considered as relevant inconsistency between studies. Statistical analyses were performed using Stata software. ${ }^{17}$

\section{RESULTS}

Of the 3281 papers, 180 potentially relevant full-text articles were independently reviewed. From these, 22 studies $(<1 \%)$ were identified as relevant for our review. Figure 1 depicts the Preferred Reporting Items for Systematic reviews and Meta-analysis flowchart. Consultation of the ENCePP register and other websites did not identify additional studies of interest.

Table, Supplemental Digital Content 3, http://links.lww.com/ INF/D740 shows the main characteristics of the 22 postlicensure observational studies meeting the inclusion criteria. Studies were published from 2012 to 2019. Fourteen studies were conducted in Europe [Denmark and Sweden (5), Finland (1), France (3), Norway (1), the Netherlands (1) and UK (3)] and 8 in North America [Canada (2) and USA (6)]. Most studies used a retrospective study design and a variety of types of data sources: registers in Nordic countries, general practitioners and hospital databases in UK and Canada, and claims databases in France and USA. Two prospective studies were conducted in France by using the Pharmacoepidemiologic General Research Extension methodology, a research platform recruiting prospectively and routinely autoimmune disorder cases through a network of specialists. Most studies included females only with ages ranging from 9 to 44 years, 1 study included males only, and 2 studies included males and females of any age. Various types of study designs were implemented to assess the risk of autoimmune diseases after HPV vaccination; 3 case-control studies including 1 matched, ${ }^{18} 1$ unmatched $^{19}$ and 1 nested case-control ${ }^{20}$; 7 case-only designs including 5 self-controlled case series ${ }^{21-25}$ and 2 case-centered method ${ }^{26,27}$; and 12 cohort studies ${ }^{8,9,28-37}$ including a surveillance study. ${ }^{8}$ More than 60 different autoimmune and rare adverse events were studied. Some studies focused on any autoimmune diseases $(n=4))^{28,31,32,35}$ others $(n=18)^{8,9,18-27,29,30,33,34,36,37}$ targeted specific outcomes. Type 1 diabetes mellitus was the most frequently studied (in 11 studies)..$^{8,9,18,19,24,28,31-33,35,37}$ Autoimmune 


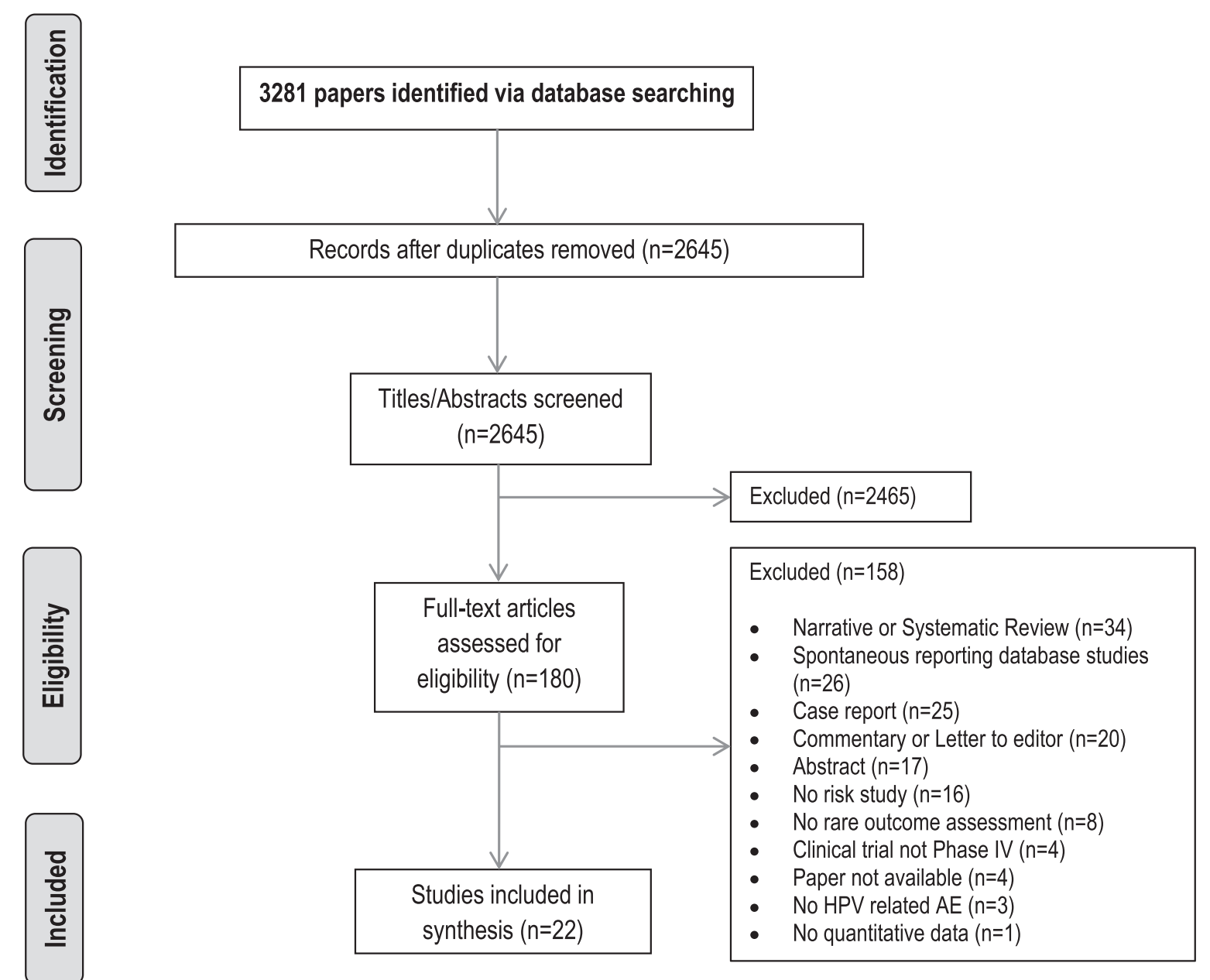

$\mathrm{HPV}=$ Human Papillomavirus Vaccine, $\mathrm{AE}=$ Adverse Event

FIGURE 1. PRISMA flowchart of the selection procedure. PRISMA indicates Preferred Reporting Items for Systematic reviews and Meta-analysis.

thyroiditis diseases $8,9,19,24,28,32,35,37$ including Hashimoto and Grave diseases, hypothyroidism and other hyperthyroidism, and immune thrombocytopenia purpura $8,9,18,19,24,28,32,35$ (ITP) were assessed in 8 studies: Crohn disease $21,24,28,32,35,37$ in 6 studies, Bell palsy, , $^{24,28,31,32,35}$ coeliac disease, $, 928,31,32,35$ juvenile rheumatoid arthritis, $8,9,24,35$ optic neuritis, ${ }^{8,24,27,28,36}$ rheumatoid arthritis, ${ }^{8,9,28,31,32}$ ulcerative colitis $^{24,28,31,32,35}$ and systemic lupus erythematosus ${ }^{8,9,28,32,35}$ in 5 studies; acute disseminated encephalomyelitis, ${ }^{8,20,24,26}$ chronic fatigue syndrome, ${ }^{22,25,30,35}$ epilepsy, ${ }^{28,31,32,35}$ Henoch-Schonlein purpura, ${ }^{28,31,32,35}$ pancreatitis, ${ }^{9,28,32,35}$ paralysis, ${ }^{28,31,32,35}$ psoriasis, ${ }^{28,31,32,35}$ vasculitis, ${ }^{9,28,31,32}$ venous thrombocytopenia ${ }^{23,28,31,35}$ and vitiligo ${ }^{28,31,32,35}$ in 4 studies; ankylosing spondylitis, ${ }^{28,31,32}$ erythema nodosum, ${ }^{28,32,35}$ hemolytic anemia, ${ }^{8,24,32}$ multiple sclerosis, ${ }^{8,20,34}$ myositis, ${ }^{28,32,35}$ narcolepsy, ${ }^{28,31,32}$ Raynaud disease, ${ }^{28,32,35}$ scleroderma $^{9,28,32}$ in 3 studies. Four studies ${ }^{9,19,21,29}$ among 7 including data on GuillainBarre syndrome (GBS) provided risk estimates. Fourteen studies $^{8,18,20,23,24,26-32,34,36}$ concerned $4 \mathrm{vHPV}$ vaccine exposure, while 4 studies $^{22,25,35,37}$ assessed $2 \mathrm{vHPV}$ vaccine exposure. Two studies ${ }^{9,21}$ provided risk estimates separately for $4 \mathrm{vHPV}$ and $2 \mathrm{vHPV}$ vaccine exposures. Two studies ${ }^{19,33}$ assessed a combined exposure to both $2 \mathrm{v}$-and $4 \mathrm{vHPV}$ and both $4 \mathrm{vHPV}$ and $9 \mathrm{vHPV}$ vaccines.

Based on the adapted quality checklist, the 22 studies included in this review were considered to have a satisfactory methodologic quality. The quality assessment scores for each study are reported in Table, Supplemental Digital Content 3, http://links. lww.com/INF/D740.

\section{Methodologic Approaches}

Methodologic considerations of the 22 studies including methods for identification and validation of cases, diagnostic criteria, onset of the diseases and analytical parameters are further detailed in Table, Supplemental Digital Content 4, http://links.lww. com/INF/D741.

\section{Definition of Outcome}

Five studies ${ }^{8,9,20,27,37}$ developed complex algorithms combining diagnosis codes and additional clinical information such as medications, laboratory test results and referral to specialists to identify cases. Fifteen studies ${ }^{19,21-26,28-36}$ identifying cases by diagnosis codes only implemented a review of all medical charts or contacted health-care providers ( 6 studies). ${ }^{21,24,26,27,33,36}$ Three studies, ${ }^{8,20,37}$ in addition to elaborated algorithms, put in place a case ascertainment process with a panel of specialist physicians. In most of the studies, the disease onset was the date of the first diagnosis, whereas in 2 studies ${ }^{20,37}$ using case ascertainment process, the criteria of first clinical sign or symptom were used. 


\section{Analytical Parameters}

In some studies, ${ }^{18,19,24,34,37}$ the researchers created composite endpoints including multiple disease conditions such as for demyelinating diseases, connective tissue disorders or neuroinflammatory diseases. Two studies ${ }^{24,37}$ analyzed composite endpoints as primary objective, while all other studies assessed individual endpoint as their primary objective. Several time frames, most often disease-specific, were evaluated in case-control designs ranging from 14 days until 3 years before disease onset. In cohort designs, the follow-up time varied from 180 days until 10 years after the last exposure irrespective of the disease. Case-only study designs were usually applied for individual endpoints only. In those studies, risk windows were defined according to the disease varying from 42 days to 1 year after disease onset. Detailed risk windows are presented in Table, Supplemental Digital Content 4, http://links. lww.com/INF/D741.

\section{Risk Estimates}

We computed pooled risk estimates for the following categories of autoimmune diseases: dermatologic (including erythema nodosum, psoriasis, scleroderma, systemic lupus erythematosus and vitiligo); hematologic (including ITP); gastrointestinal (including coeliac, Crohn disease, pancreatitis and ulcerative colitis); musculoskeletal or systemic diseases (including ankylosing spondylitis, Henoch-Schonlein purpura, juvenile rheumatoid arthritis, myositis, rheumatoid arthritis and vasculitis); neurologic (Bell palsy, epilepsy, GBS, chronic fatigue syndrome, narcolepsy and paralysis); ophthalmic (optic neuritis); other demyelinating diseases (including central nervous system disorders and multiple sclerosis); thyroiditis disorders (including Hashimoto, Graves' disease and other hyperthyroidism); other disorders (including Raynaud disease, Sjogren syndrome and venous thrombocytopenia) and type 1 diabetes. Pooled estimates were computed for 35 disease conditions corresponding to 48 pooled estimates. Pooled estimates are reported in Table 2. Risk estimates for all autoimmune diseases and other rare events are reported in Table, Supplemental Digital Content 5, http://links.lww.com/INF/D742. Most of them were computed for $4 \mathrm{vHPV}(\mathrm{n}=34)$ and the remaining for $2 \mathrm{vHPV}(\mathrm{n}=14)$. Majority of the pooled estimates did not show significant association $(\mathrm{n}=$ 43). Three pooled estimates showed a protective effect for ITP [OR $=0.55(95 \% \mathrm{CI}: 0.34-0.88)]$ and chronic fatigue syndrome [OR $=0.77(95 \% \mathrm{CI}: 0.62-0.97)]$ after $2 \mathrm{vHPV}$ vaccine and for paralysis $[\mathrm{OR}=0.52(95 \% \mathrm{CI}: 0.55-0.77)]$ after $4 \mathrm{vHPV}$ vaccine. Two pooled estimates showed a statistically significant increased risk for Hashimoto disease [OR $=1.25$ (95\% CI: $1.09-1.44)]$ and Raynaud disease $[\mathrm{OR}=1.63(95 \% \mathrm{CI}: 1.21-2.20)]$ after 4vHPV vaccine.

For diseases reported in a single study, 9 risk estimates showed statistically significant associations (Table, Supplemental Digital Content 5, http://links.lww.com/INF/D742). Increased risks with relatively large $95 \%$ CI were observed in males following 9vHPV for narcolepsy [RR $=3.44$ (95\% CI: 1.08-11.0)] and vitiligo $[\mathrm{RR}=4.70(95 \% \mathrm{CI}: 1.13-19.5)]$.

\section{DISCUSSION AND CONCLUSIONS}

Following large-scale use of HPV vaccines, rare serious adverse events have been reported which prompted additional investigations. ${ }^{38-40}$ Further to this, several postlicensure studies were conducted to estimate associations between HPV vaccination and autoimmune and other rare adverse events. The present review is to our knowledge the first comprehensive review aiming to describe the methodologic approaches used in HPV vaccine postlicensure observational studies. In addition, we aimed to summarize risk estimates of autoimmune and other rare events following immunization with HPV from the available evidence. Among the 22 postlicensure observational safety studies included, we identified 2 important elements informing on the validity and robustness of postlicensure studies assessing rare adverse events.

The first element is related to the validity of the clinical outcomes and determination of onset of disease. In the eligible studies, simple to more complex algorithms were developed to identify and validate cases of autoimmune diseases. The level of granularity of the clinical case definition may generate, if not consistent across studies, an important source of heterogeneity restricting direct comparison between studies. As an example, 1 study $^{9}$ broadly defined autoimmune thyroiditis diseases including in its definition codes for disease of nonautoimmune origin, while other studies $8,24,28,32,35$ targeted specific medical conditions such as Hashimoto disease. In addition, algorithm-based approach should ensure a high specificity of the outcome definition and therefore avoid inclusion of false-positive subjects. Algorithm-based search only is deemed sufficiently robust to detect acute events such as GBS. However, for diseases with insidious onset, such approach may introduce bias on the true onset date.

Second important element is related to the analytical parameters including risk period, endpoints and sample size. While a long and sufficient follow-up time is required for long latency diseases to be detected, risk period must be adequately defined to establish accurate evidence of a causal relationship. Some autoimmune events are known to occur within few weeks after vaccine exposure such as GBS detected between 6 and 8 weeks after swine flu vaccine ${ }^{41,42}$ For some other autoimmune diseases, evidence of time to disease onset (ie, multiple sclerosis) ${ }^{43}$ or lag time between onset of symptoms and disease diagnosis (ie, rheumatoid arthritis) ${ }^{44}$ are not well clearly established. In such circumstances, risk periods should be defined as much as possible using epidemiologic and mechanistic evidences or by expert opinion. Sensitivity analyses using different risk periods and clustering analyses are complementary methods to highlight potential windows of risk. A disease-specific time frame should be the preferred approach when different kind of clinical events are under assessment. In case-only study design, a washout period between risk and control periods should also be preferably considered, to avoid misclassification. ${ }^{45}$ In the studies, sample sizes limitations were overcome by creating composite endpoints or by combining multiple healthcare databases. With intrinsic limitations such as heterogeneity between databases or lack of specificity of the outcome of interest, both alternative approaches emphasize the need for collaboration to increase sample size and develop common clinical definitions. Because of the rarity of autoimmune diseases, some of the studies included in our review may not be adequately powered to detect a potential increased or decreased risk. This possible lack of statistical power may suggest an unreliable absence of risk. In addition, 2 vHPV vaccine was less frequently studied and therefore no estimation of risk could be generated for some of the diseases.

Our review also provides pooled risk estimates. Pooled findings in females suggested that $4 \mathrm{vHPV}$ vaccination significantly increased the risk of both Hashimoto and Raynaud diseases. The slightly elevated risk of Hashimoto disease was mainly driven by the Chao et $\mathrm{al}^{8}$ study. After further evaluation, the researchers demonstrated that most of the new onset cases were likely preexisting cases and that no consistent evidence for a safety signal for autoimmune thyroid conditions in general was observed among vaccinated subjects. Similarly, the increased risk of Raynaud disease was driven by the Arnheim-Dahlström et al ${ }^{28}$ study. The researchers discriminated this safety signal based on prespecified causality criteria including the strength of the association (rate ratio $<3.0$ ). On the contrary, a protective effect was observed for paralysis after $4 \mathrm{vHPV}$ and for ITP and chronic fatigue syndrome after $2 \mathrm{vHPV}$. By 
TABLE 2. Summary of Pooled Estimates (ORs) for 35 Autoimmune Diseases and/or Other Rare Adverse Events

\begin{tabular}{|c|c|c|c|}
\hline Outcomes & $\begin{array}{l}\text { HPV Vaccine } \\
\text { Exposure }\end{array}$ & $\begin{array}{l}\text { Pooled Estimates* } \\
\text { [OR }(95 \% \mathrm{CI})]\end{array}$ & $\begin{array}{c}I^{2} \text { Statistics } \\
(\%)\end{array}$ \\
\hline \multicolumn{4}{|l|}{ Dermatologic } \\
\hline Erythema nodosum & $4 \mathrm{vHPV}$ & $1.26(0.89 ; 1.79)$ & 0.0 \\
\hline Psoriasis & $4 \mathrm{vHPV}$ & $1.03(0.87 ; 1.23)$ & 0.0 \\
\hline Scleroderma & $4 \mathrm{vHPV}$ & $1.04(0.64 ; 1.69)$ & 29.7 \\
\hline \multirow[t]{2}{*}{ Systemic lupus erythematosus } & $4 \mathrm{vHPV}$ & $1.04(0.82 ; 1.33)$ & 0.0 \\
\hline & $2 \mathrm{vHPV}$ & $1.20(0.39 ; 3.68)$ & 20.5 \\
\hline Vitiligo & $4 \mathrm{vHPV}$ & $1.31(0.91 ; 1.87)$ & 25.6 \\
\hline \multicolumn{4}{|l|}{ Diabetes } \\
\hline \multirow{2}{*}{ Diabetes type 1 diabetes mellitus } & $4 \mathrm{vHPV}$ & $0.93(0.65 ; 1.34)$ & 86.5 \\
\hline & $2 \mathrm{vHPV}$ & $0.80(0.50 ; 1.26)$ & 58.5 \\
\hline \multicolumn{4}{|l|}{ Hematologic } \\
\hline \multirow{2}{*}{ Idiopathic thrombocytopenia purpura } & $4 \mathrm{vHPV}$ & $1.06(0.85 ; 1.33)$ & 40.2 \\
\hline & $2 \mathrm{vHPV}$ & $0.55(0.34 ; 0.88)$ & 0.0 \\
\hline \multicolumn{4}{|l|}{ Gastrointestinal } \\
\hline \multirow{2}{*}{ Coeliac disease } & $4 \mathrm{vHPV}$ & $1.16(0.87 ; 1.56)$ & 67.3 \\
\hline & $2 \mathrm{vHPV}$ & $1.05(0.80 ; 1.38)$ & 0.0 \\
\hline \multirow[t]{2}{*}{ Crohn disease } & $4 \mathrm{vHPV}$ & $1.04(0.73 ; 1.47)$ & 69.3 \\
\hline & $2 \mathrm{vHPV}$ & $1.17(0.77 ; 1.78)$ & 0.0 \\
\hline \multirow[t]{2}{*}{ Pancreatitis } & $4 \mathrm{vHPV}$ & $0.87(0.69 ; 1.08)$ & 0.0 \\
\hline & $2 \mathrm{vHPV}$ & $1.68(0.85 ; 3.33)$ & 0.0 \\
\hline \multirow[t]{2}{*}{ Ulcerative colitis } & $4 \mathrm{vHPV}$ & $0.93(0.58 ; 1.50)$ & 80.7 \\
\hline & $2 \mathrm{vHPV}$ & $0.57(0.15 ; 2.23)$ & 80.7 \\
\hline \multicolumn{4}{|l|}{ Musculoskeletal/systemic } \\
\hline Ankylosing spondylitis & $4 \mathrm{vHPV}$ & $0.98(0.65 ; 1.48)$ & 0.0 \\
\hline Behcet syndrome & $4 \mathrm{vHPV}$ & $1.52(0.29 ; 7.96)$ & 69.4 \\
\hline Henoch-Schonlein purpura & $4 \mathrm{vHPV}$ & $1.03(0.66 ; 1.60)$ & 0.0 \\
\hline \multirow{2}{*}{ Juvenile rheumatoid arthritis } & 4vHPV & $0.73(0.36 ; 1.47)$ & 77.7 \\
\hline & $2 \mathrm{vHPV}$ & $1.03(0.82 ; 1.29)$ & 6.2 \\
\hline Myositis & $4 \mathrm{vHPV}$ & $0.92(0.50 ; 1.69)$ & 0.0 \\
\hline Polymyositis/dermatomyositis & $4 \mathrm{vHPV}$ & $0.83(0.46 ; 1.51)$ & 0.0 \\
\hline Rheumatoid arthritis & $4 \mathrm{vHPV}$ & $0.92(0.72 ; 1.17)$ & 0.0 \\
\hline Vasculitis & $4 \mathrm{vHPV}$ & $1.11(0.86 ; 1.42)$ & 0.0 \\
\hline \multicolumn{4}{|l|}{ Neurologic } \\
\hline \multirow{2}{*}{ Bell palsy } & $4 \mathrm{vHPV}$ & $0.79(0.46 ; 1.35)$ & 73.8 \\
\hline & $2 \mathrm{vHPV}$ & $1.37(0.83 ; 2.26)$ & 0.0 \\
\hline Epilepsy & $4 \mathrm{vHPV}$ & $0.81(0.54 ; 1.24)$ & 87.7 \\
\hline \multirow{2}{*}{ Guillain-Barre syndrome } & $4 \mathrm{vHPV}$ & $1.79(0.65 ; 4.94)$ & 64.0 \\
\hline & $2 \mathrm{vHPV}$ & $2.89(0.58 ; 14.40)$ & 69.7 \\
\hline Chronic fatigue syndrome & $2 \mathrm{vHPV}$ & $0.77(0.62 ; 0.97)$ & 11.5 \\
\hline Narcolepsy & $4 \mathrm{vHPV}$ & $1.08(0.64 ; 1.84)$ & 19.3 \\
\hline Paralysis & $4 \mathrm{vHPV}$ & $0.52(0.35 ; 0.77)$ & 0.0 \\
\hline \multicolumn{4}{|l|}{ Ophthalmic } \\
\hline Optic neuritis & $4 \mathrm{vHPV}$ & $1.20(0.84 ; 1.71)$ & 19.8 \\
\hline \multicolumn{4}{|l|}{ Other demyelinating diseases } \\
\hline $\begin{array}{l}\text { Central nervous system demyelinating } \\
\text { syndrome }\end{array}$ & $4 \mathrm{vHPV}$ & $1.02(0.77 ; 1.33)$ & 0.0 \\
\hline Multiple sclerosis & $4 \mathrm{vHPV}$ & $0.96(0.77 ; 1.21)$ & 0.0 \\
\hline Other disorders & & & \\
\hline Raynaud disease & 4vHPV & $1.63(1.21 ; 2.20)$ & 0.0 \\
\hline Sjogren syndrome & $4 \mathrm{vHPV}$ & $1.34(0.71 ; 2.51)$ & 0.0 \\
\hline Venous thrombocytopenia & $4 \mathrm{vHPV}$ & $0.80(0.60 ; 1.07)$ & 0.0 \\
\hline Thyroid & & & \\
\hline Hashimoto disease & $4 \mathrm{vHPV}$ & $1.25(1.09 ; 1.44)$ & 0.0 \\
\hline & $2 \mathrm{vHPV}$ & $0.88(0.57 ; 1.36)$ & 0.0 \\
\hline Grave disease & $4 \mathrm{vHPV}$ & $0.88(0.73 ; 1.07)$ & 3.7 \\
\hline & $2 \mathrm{vHPV}$ & $1.12(0.56 ; 2.24)$ & 63.3 \\
\hline Autoimmune thyroiditis & $4 \mathrm{vHPV}$ & $1.10(0.94 ; 1.27)$ & 0.0 \\
\hline & $2 \mathrm{vHPV}$ & $1.76(0.65 ; 4.77)$ & 83.3 \\
\hline Other hyperthyroidism & $4 \mathrm{vHPV}$ & $0.98(0.79 ; 1.22)$ & 0.0 \\
\hline
\end{tabular}

Bold estimates are statistically significant.*Pooled estimates were computed using fixed-effects model or randomeffects model $\left(I^{2}>50 \%\right)$ in Stata v14.0.

using criteria such as the strength of the association, the consistency of the reported risks and the level of significance to interpret the risk estimates, we noticed a lack of clear association for all protective and risk effects with regard to HPV vaccines. In addition, the pooled analysis has several limitations due to heterogeneity of clinical definitions, targeted age categories and variation in risk periods across studies. Two studies ${ }^{28,32}$ conducted in Sweden and Denmark used same sources of data but targeted 2 different age categories (10-17 and 18-44 years old). Therefore, the pooled estimate for Raynaud disease and paralysis does not bring any added 
value and only the individual studies can confirm the observed risks. Similarly, a higher risk of Hashimoto disease was observed from a pool of 3 studies for which the age of the populations did not necessarily overlap. Moreover, the small number of studies included in our analysis did not allow stratification by type of study design or risk estimates. The pooling of any risk estimates did not affect drastically the pooled estimates because for rare events OR and relative risk are virtually similar. However, while each individual study was fit-for-purpose for the stated objectives, the variability of methods across studies may impact the ability to validly characterize risks. The risk evaluation in our review should be considered as an indicator of possible harms after HPV vaccination for which enhanced and continuous surveillance should be maintained or implemented. A recently published meta-analysis ${ }^{11}$ also showed a small increased risk of Hashimoto disease after HPV vaccination. However, the authors did not provide analysis by type of HPV vaccine. Because the mechanisms of action of adjuvant systems may perform differently, a critical evaluation by type of vaccine may help to discriminate any potential triggering effect.

This review also underlines the need for harmonization of outcome definitions and collaboration in assessing vaccine safety, which is one of the efforts currently done in the ADVANCE project ${ }^{46}$ in the European Union and was possible globally for assessing safety of the pandemic influenza vaccine. ${ }^{47}$

In conclusion, this systematic review emphasizes the diversity of methodologic approaches to assess the risk of developing rare adverse events after HPV vaccination. Results show that many events have been studied but not systematically for the different HPV vaccines. The review highlights that positive and negative associations were observed with autoimmune diseases. However, these estimates should be interpreted with caution due to the diversity in methodologic approaches used by the studies included in this review. More systematic collaborations and harmonization of event clinical definitions are needed to monitor rare safety events.

\section{ACKNOWLEDGMENT}

The authors would like to thank Sabine Straus and Caitlin Dodd for their critical review of this manuscript.

\section{REFERENCES}

1. Mesher D, Panwar K, Thomas SL, et al. Continuing reductions in HPV $16 / 18$ in a population with high coverage of bivalent HPV vaccination in England: an ongoing cross-sectional study. BMJ Open. 2016;6:e009915.

2. Herweijer E, Sundström K, Ploner A, et al. Quadrivalent HPV vaccine effectiveness against high-grade cervical lesions by age at vaccination: a population-based study. Int J Cancer. 2016;138:2867-2874.

3. Kash N, Lee MA, Kollipara R, et al. Safety and efficacy data on vaccines and immunization to human papillomavirus. J Clin Med. 2015;4: 614-633.

4. Haupt RM, Sings HL. The efficacy and safety of the quadrivalent human papillomavirus 6/11/16/18 vaccine gardasil. J Adolesc Health. 2011;49: 467-475.

5. Gallagher KE, LaMontagne DS, Watson-Jones D. Status of HPV vaccine introduction and barriers to country uptake. Vaccine. 2018;36(32 Pt A):4761-4767.

6. Bruni L, Diaz M, Barrionuevo-Rosas L, et al. Global estimates of human papillomavirus vaccination coverage by region and income level: a pooled analysis. Lancet Glob Health. 2016;4:e453-e463.

7. Batista-Duharte A, Martínez DT, Carlos IZ. Efficacy and safety of immunological adjuvants. Where is the cut-off? Biomed Pharmacother. 2018; 105:616-624.

8. Chao C, Klein NP, Velicer CM, et al. Surveillance of autoimmune conditions following routine use of quadrivalent human papillomavirus vaccine. J Intern Med. 2012;271:193-203.

9. Miranda S, Chaignot C, Collin C, et al. Human papillomavirus vaccination and risk of autoimmune diseases: a large cohort study of over 2 million young girls in France. Vaccine. 2017;35:4761-4768.
10. Genovese C, LA Fauci V, Squeri A, et al. HPV vaccine and autoimmune diseases: systematic review and meta-analysis of the literature. J Prev Med Hyg. 2018;59:E194-E199.

11. Jiang HY, Shi YD, Zhang X, et al. Human papillomavirus vaccination and the risk of autoimmune disorders: a systematic review and meta-analysis. Vaccine. 2019;37:3031-3039.

12. Moher D, Liberati A, Tetzlaff J, et al.; PRISMA Group. Preferred reporting items for systematic reviews and meta-analyses: the PRISMA statement. $J$ Clin Epidemiol. 2009;62:1006-1012.

13. The European Network of Centres for Pharmacoepidemiology and Pharmacovigilance (ENCePP) Guide on Methodological Standards in Pharmacoepidemiology (Revision 6). EMA/95098/2010. Available from: http://www.encepp.eu/standards_and_guidances. Accessed June 07, 2019.

14. The European Union electronic register of post-authorisation studies (EU PAS Register). Available from: http://www.encepp.eu/encepp_studies/ indexRegister.shtml. Accessed June 07, 2019.

15. Wells GA, Shea B, O'Connell O, et al: The Newcastle-Ottawa Scale (NOS) for assessing the quality of nonrandomised studies in meta-analyses. Available from: http://www.ohri.ca/programs/clinical_epidemiology/ oxford.asp. Accessed June 07, 2019].

16. Scottish Intercollegiate Guidelines Network. Critical appraisal: notes and checklists. Available from: http:/www.sign.ac.uk/checklists-and-notes. html. Accessed June 07, 2019.

17. StataCorp.Stata Statistical Software: Release 14. College Station, TX: StataCorp LP; 2015.

18. Grimaldi-Bensouda L, Guillemot D, Godeau B, et al.; PGRx-AID Study Group. Autoimmune disorders and quadrivalent human papillomavirus vaccination of young female subjects. J Intern Med. 2014;275:398-408.

19. Grimaldi-Bensouda L, Rossignol M, Koné-Paut I, et al.; PGRx-AD Study Group. Risk of autoimmune diseases and human papilloma virus (HPV) vaccines: Six years of case-referent surveillance. J Autoimmun. 2017;79: 84-90.

20. Langer-Gould A, Qian L, Tartof SY, et al. Vaccines and the risk of multiple sclerosis and other central nervous system demyelinating diseases. JAMA Neurol. 2014;71:1506-1513.

21. Andrews N, Stowe J, Miller E. No increased risk of Guillain-Barré syndrome after human papilloma virus vaccine: a self-controlled case-series study in England. Vaccine. 2017;35:1729-1732.

22. Donegan K, Beau-Lejdstrom R, King B, et al. Bivalent human papillomavirus vaccine and the risk of fatigue syndromes in girls in the UK. Vaccine. 2013;31:4961-4967.

23. Scheller NM, Pasternak B, Svanström H, et al. Quadrivalent human papillomavirus vaccine and the risk of venous thromboembolism. JAMA. 2014;312:187-188.

24. Liu EY, Smith LM, Ellis AK, et al. Quadrivalent human papillomavirus vaccination in girls and the risk of autoimmune disorders: the Ontario Grade 8 HPV Vaccine Cohort Study. CMAJ. 2018;190:E648-E655.

25. Schurink-Van't Klooster TM, Kemmeren JM, van der Maas NAT, et al No evidence found for an increased risk of long-term fatigue following human papillomavirus vaccination of adolescent girls. Vaccine. 2018;36: 6796-6802.

26. Baxter R, Lewis E, Goddard K, et al. Acute demyelinating events following vaccines: a case-centered analysis. Clin Infect Dis. 2016;63:1456-1462.

27. Baxter R, Lewis E, Fireman B, et al. Case-centered analysis of optic neuritis after vaccines. Clin Infect Dis. 2016;63:79-81.

28. Arnheim-Dahlström L, Pasternak B, Svanström H, et al. Autoimmune, neurological, and venous thromboembolic adverse events after immunisation of adolescent girls with quadrivalent human papillomavirus vaccine in Denmark and Sweden: cohort study. BMJ. 2013;347:f5906.

29. Deceuninck G, Sauvageau C, Gilca V, et al. Absence of association between Guillain-Barré syndrome hospitalizations and HPV-vaccine. Expert Rev Vaccines. 2018;17:99-102.

30. Feiring B, Laake I, Bakken IJ, et al. HPV vaccination and risk of chronic fatigue syndrome/myalgic encephalomyelitis: a nationwide register-based study from Norway. Vaccine. 2017;35:4203-4212.

31. Frisch M, Besson A, Clemmensen KKB, et al. Quadrivalent human papillomavirus vaccination in boys and risk of autoimmune diseases, neurological diseases and venous thromboembolism. Int J Epidemiol. 2018;47: 634-641.

32. Hviid A, Svanström H, Scheller NM, et al. Human papillomavirus vaccination of adult women and risk of autoimmune and neurological diseases. $J$ Intern Med. 2018;283:154-165. 
33. Klein NP, Goddard K, Lewis E, et al. Long term risk of developing type 1 diabetes after HPV vaccination in males and females. Vaccine. 2019;37:1938-1944.

34. Scheller NM, Svanström H, Pasternak B, et al. Quadrivalent HPV vaccination and risk of multiple sclerosis and other demyelinating diseases of the central nervous system. JAMA. 2015;313:54-61.

35. Skufca J, Ollgren J, Artama M, et al. The association of adverse events with bivalent human papilloma virus vaccination: a nationwide register-based cohort study in Finland. Vaccine. 2018;36:5926-5933.

36. Sridhar G, Tian F, Forshee R, et al. Evaluation of optic neuritis following human papillomavirus vaccination. Hum Vaccin Immunother. 2017;13:1705-1713.

37. Willame C, Rosillon D, Zima J, et al. Risk of new onset autoimmune disease in 9- to 25-year-old women exposed to human papillomavirus-16/18 AS04-adjuvanted vaccine in the United Kingdom. Hum Vaccin Immunother. 2016;12:2862-2871.

38. Colafrancesco S, Perricone C, Tomljenovic L, et al. Human papilloma virus vaccine and primary ovarian failure: another facet of the autoimmune/ inflammatory syndrome induced by adjuvants. Am J Reprod Immunol. 2013;70:309-316.

39. Kinoshita T, Abe RT, Hineno A, et al. Peripheral sympathetic nerve dysfunction in adolescent Japanese girls following immunization with the human papillomavirus vaccine. Intern Med. 2014;53:2185-2200.
40. European Medicines Agency. EMA to further clarify safety profile of human papillomavirus (HPV) vaccines. July 2015. Available from: http://www. ema.europa.eu/docs/en_GB/document_library/Press_release/2015/07/ WC500189481.pdf. Accessed June 07, 2019.

41. Schonberger LB, Bregman DJ, Sullivan-Bolyai JZ, et al. Guillain-Barre syndrome following vaccination in the national influenza immunization program, United States, 1976-1977. Am J Epidemiol. 1979;110:105-123.

42. Wise ME, Viray M, Sejvar JJ, et al. Guillain-Barre syndrome during the 20092010 H1N1 influenza vaccination campaign: population-based surveillance among 45 million Americans. Am J Epidemiol. 2012;175:1110-1119.

43. Institute of Medicine. Adverse Effects of Vaccines: Evidence and Causality. Washington, DC: The National Academies Press; 2012.

44. Chan KW, Felson DT, Yood RA, et al. The lag time between onset of symptoms and diagnosis of rheumatoid arthritis. Arthritis Rheum. 1994;37:814-820.

45. Li R, Stewart B, Weintraub E. Evaluating efficiency and statistical power of self-controlled case series and self-controlled risk interval designs in vaccine safety. J Biopharm Stat. 2016;26:686-693.

46. ADVANCE. Available from: http://www.advance-vaccines.eu/. Accessed June 07, 2019.

47. Dodd CN, Romio SA, Black S, et al.; Global H1N1 GBS Consortium. International collaboration to assess the risk of Guillain Barré syndrome following Influenza A (H1N1) 2009 monovalent vaccines. Vaccine. 2013;31:4448-4458 